\title{
LOCAL UNIFORM LINEAR CONVEXITY WITH RESPECT TO THE KOBAYASHI DISTANCE
}

\author{
MONIKA BUDZYŃSKA
}

Received 2 October 2001

We introduce the notion of local uniform linear convexity of bounded convex domains with respect to their Kobayashi distances.

\section{Introduction}

Recently, in [4] the author has proved that if $B$ is an open unit ball in a Cartesian product $l^{2} \times l^{2}$ furnished with the $l^{p}$-norm $\|\cdot\|$ and $k_{B}$ is the Kobayashi distance on $B$, then the metric space $\left(B, k_{B}\right)$ is locally uniformly linearly convex.

In this paper, we introduce this kind of local uniform convexity in bounded convex domains in complex reflexive Banach spaces and we apply this notion in the fixed-point theory of holomorphic mappings.

\section{Preliminaries}

Throughout this paper all Banach spaces $X$ will be complex and reflexive and all domains $D \subset X$ will be bounded and convex. By $k_{D}$ we always denote the Kobayashi distance on $D[16,17]$ (see also $[10,12,14,15,19]$ ).

We now recall several useful properties of the Kobayashi distance $k_{D}$, which are common to all bounded and convex domains in reflexive Banach spaces.

Since $D$ is bounded and convex, the Kobayashi and Caratheodory distances are equal on $D$ [7]. The Kobayashi distance $k_{D}$ is locally equivalent to the norm [14].

If $x, y, w, z \in D$ and $s \in[0,1]$, then

$$
k_{D}(s x+(1-s) y, s w+(1-s) z) \leq \max \left\{k_{D}(x, w), k_{D}(y, z)\right\}
$$

Hence each open (closed) $k_{D}$-ball in the metric space $\left(B, k_{B}\right)$ is convex [20]. 
A subset $C$ of $D$ is said to lie strictly inside $D$ if $\operatorname{dist}_{\|\cdot\|}(C, \partial D)>0$.

The basic fact about subsets, which lie strictly inside $D$, is the following: a subset $C$ of $D$ is $k_{D}$-bounded if and only if $C$ lies strictly inside $D$ ([14, Proposition 23]).

A point $x$ on the boundary of a convex set $D \in X$ is called an extreme point if $\{x+t y \in X:-1 \leq t \leq 1\} \subset \bar{D}$ implies $y=0$. If each boundary point of a convex domain $D$ is an extreme point, then $D$ is called a strictly convex domain. If $D$ is strictly convex, then we can say more about linear convexity of balls in $\left(D, k_{D}\right)$. In this case, each $k_{D}$-ball is also strictly convex in linear sense $[5,23]$ (see also [22]).

The open unit ball $B_{H}$ in a Hilbert space is called the Hilbert ball $[6,12,13$, 21].

\section{Local uniform linear convexity for the Kobayashi distance}

First, we introduce the following definition (see also $[4,18]$ ).

Definition 3.1. Let $D$ be a bounded and convex domain in a reflexive Banach space $X$. The metric space $\left(D, k_{D}\right)$ is said to be a locally uniformly linearly convex space if there exist $w \in D$ and the function

$$
\delta(w, \cdot, \cdot, \cdot, \cdot, \cdot),
$$

such that for all $0<R_{1}, k_{D}(w, z) \leq R_{1}, 0<R_{2} \leq R \leq R_{3}$, and $0<\epsilon_{1} \leq \epsilon \leq \epsilon_{2}<2$, we have

$$
\left.\begin{array}{l}
\quad \delta\left(w, R_{1}, R_{2}, R_{3}, \epsilon_{1}, \epsilon_{2}\right)>0 \\
k_{D}(z, x) \leq R \\
k_{D}(z, y) \leq R \\
k_{D}(x, y) \geq \epsilon R
\end{array}\right\} \Longrightarrow k_{D}\left(z, \frac{1}{2} x+\frac{1}{2} y\right) \leq\left(1-\delta\left(w, R_{1}, R_{2}, R_{3}, \epsilon_{1}, \epsilon_{2}\right)\right) R
$$

The function $\delta(w, \cdot, \cdot, \cdot, \cdot, \cdot)$ is called a modulus of linear convexity for the Kobayashi distance $k_{D}$.

It is easy to observe that the point $w$ in the above definition of the local uniform linear convexity can be replaced by any other point $w^{\prime} \in D$.

The Hilbert ball $B_{H}$ is the first known domain with this property [18] (see also [19]). Moreover, in [4] it is shown that if $B$ is the open unit ball in a Cartesian product $l^{2} \times l^{2}$ furnished with the $l^{p}$-norm where $1<p<\infty$ and $p \neq 2$, then the metric space $\left(B, k_{B}\right)$ is also locally uniformly linearly convex.

The construction of domains which are locally uniformly convex in linear sense in the Kobayashi distance is given in [3]. 


\section{Fixed points of holomorphic mappings}

We begin this section by recalling some definitions.

A mapping $f: D \rightarrow D$ is $k_{D}$-nonexpansive if

$$
k_{D}(f(x), f(y)) \leq k_{D}(x, y)
$$

for all $x, y \in D$. Each holomorphic self-mapping $f: D \rightarrow D$ is $k_{D}$-nonexpansive $[6,10,12]$.

If $f: D \rightarrow D$ is $k_{D}$-nonexpansive, then for each $0<t<1$ and $a \in B$, the mapping $f_{t, a}=(1-t) a+t f$ is a contraction. Therefore, for each $x \in D$, the sequence $\left\{f_{t, a}^{n}(x)\right\}$ tends to a unique fixed point $y_{t, a}$ in $D$. Additionally, we have $\lim _{t \rightarrow 1^{-}}\left\|y_{t, a}-f_{t, a}\left(y_{t, a}\right)\right\|=0$ [8].

For $k_{D}$-nonexpansive $f: D \rightarrow D$, we call a sequence $\left\{x_{n}\right\} \subset B$ an approximating sequence if $\lim _{n} k_{D}\left(x_{n}, f\left(x_{n}\right)\right)=0$ [12].

We will also need the notion of an asymptotic center $[9,11,12]$. Let $D$ be a bounded convex domain in a reflexive Banach space $X,\left\{x_{n}\right\}$ a $k_{D}$-bounded sequence in $D$, and $C$ a nonempty, $k_{D}$-closed, and convex subset of $D$. Consider the functional $r\left(\cdot,\left\{x_{n}\right\}\right): D \rightarrow[0, \infty)$ defined by $r\left(x,\left\{x_{n}\right\}\right)=\limsup _{n \rightarrow \infty} k_{D}\left(x, x_{n}\right)$. Recall that a point $z$ in $C$ is said to be an asymptotic center of the sequence $\left\{x_{n}\right\}$ with respect to $C$ if $r\left(z,\left\{x_{n}\right\}\right)=\inf \left\{r\left(x,\left\{x_{n}\right\}\right): x \in C\right\}$. The infimum of $r\left(\cdot,\left\{x_{n}\right\}\right)$ over $C$ is called the asymptotic radius of $\left\{x_{n}\right\}$ with respect to $C$ and denoted by $r\left(C,\left\{x_{n}\right\}\right)$. We observe that the function $r\left(\cdot,\left\{x_{n}\right\}\right)$ is quasiconvex, that is,

$$
r\left((1-t) x+t y,\left\{x_{n}\right\}\right) \leq \max \left(r\left(x,\left\{x_{n}\right\}\right), r\left(y,\left\{x_{n}\right\}\right)\right)
$$

for all $x$ and $y$ in $D$ and $0 \leq t \leq 1[19,20]$.

Proposition 4.1. Let $D$ be a bounded convex domain in a reflexive Banach space $X$ such that the metric space $\left(D, k_{D}\right)$ is locally uniformly linearly convex. Then each $k_{D}$-bounded sequence $\left\{x_{n}\right\}$ in $D$ has a unique asymptotic center with respect to any nonempty, $k_{D}$-closed, and convex subset $C$ of $D$.

Proof. Fix $w \in D$ and let $\delta(w, \cdot, \cdot, \cdot, \cdot, \cdot)$ be a modulus of linear convexity for the Kobayashi distance $k_{D}$. Let $\left\{x_{n}\right\}$ be a $k_{D}$-bounded sequence in $D$. Hence the sequence $\left\{x_{n}\right\}$ lies strictly inside $D$ and therefore we have

$$
0<\sup _{n} k_{D}\left(w, x_{n}\right)+1=R_{1}<+\infty .
$$

Next, the sets

$$
C_{n}=\left\{x \in C: r\left(x,\left\{x_{n}\right\}\right) \leq r\left(C,\left\{x_{n}\right\}\right)+\frac{1}{n}\right\}
$$


are nonempty, convex, and weakly compact since the function $r\left(\cdot,\left\{x_{n}\right\}\right)$ is continuous and quasiconvex. Hence, $r\left(\cdot,\left\{x_{n}\right\}\right)$ attains its minimum in $C$. Now, all we have to show is that

$$
r\left(\frac{1}{2} x+\frac{1}{2} y,\left\{x_{n}\right\}\right)<\max \left(r\left(x,\left\{x_{n}\right\}\right), r\left(y,\left\{x_{n}\right\}\right)\right)
$$

for every $x \neq y$. To this end, let $R_{2}=\max \left(r\left(x,\left\{x_{n}\right\}\right), r\left(y,\left\{x_{n}\right\}\right)\right)$. Then for each $0<\epsilon<1$, there exists $n_{\epsilon}$ such that

$$
k_{D}\left(x, x_{n}\right) \leq R_{2}+\epsilon, \quad k_{D}\left(y, x_{n}\right) \leq R_{2}+\epsilon,
$$

for all $n \geq n_{\epsilon}$. Therefore, for $R_{3}=R_{2}+1$ and $\epsilon_{1}=\epsilon_{2}=k_{D}(x, y) /\left(R_{2}+1\right)$, we have

$$
\begin{aligned}
& k_{D}\left(\frac{1}{2} x+\frac{1}{2} y, x_{n}\right) \leq\left(1-\delta\left(w, R_{1}, R_{2}, R_{3}, \epsilon_{1}, \epsilon_{2}\right)\right)\left(R_{2}+\epsilon\right), \\
& r\left(\frac{1}{2} x+\frac{1}{2} y,\left\{x_{n}\right\}\right) \leq\left(1-\delta\left(w, R_{1}, R_{2}, R_{3}, \epsilon_{1}, \epsilon_{2}\right)\right) R_{2}<R_{2} .
\end{aligned}
$$

This completes the proof.

Now, we are ready to prove the following theorem.

Theorem 4.2. Let $D$ be a bounded convex domain in a Banach space $X$ such that the metric space $\left(D, k_{D}\right)$ is locally uniformly linearly convex and let $f: D \rightarrow D$ be a $k_{D}$-nonexpansive mapping. Then the following statements are equivalent:

(i) $f$ has a fixed point;

(ii) there exists a point $x$ in $D$ such that the sequence of iterates $\left\{f^{n}(x)\right\}$ is $k_{D^{-}}$ bounded;

(iii) the sequence of iterates $\left\{f^{n}(x)\right\}$ is $k_{D}$-bounded for all $x$ in $D$;

(iv) there exists a $k_{D}$-bounded approximating sequence $\left\{x_{n}\right\}$ for $f$;

(v) there exists a closed and $f$-invariant $k_{D}$-ball;

(vi) there exists a nonempty, closed, and convex, $k_{D}$-bounded and $f$-invariant subset $C$ of $D$.

Proof. To prove this theorem, it is sufficient to apply the asymptotic center method and the following facts:

(1) each nonempty, closed, and convex, $k_{D}$-bounded and $f$-invariant subset $C$ of $D$ contains a $k_{D}$-bounded approximating sequence for $f$;

(2) if $\left\{x_{n}\right\}$ is a $k_{D}$-bounded approximating sequence for $f$, then

$$
r\left(f(y),\left\{x_{n}\right\}\right) \leq r\left(y,\left\{x_{n}\right\}\right)
$$

for each $y \in D$; 
(3) if $x \in D$ has the $k_{D}$-bounded sequence of iterates $\left\{f^{n}(x)\right\}$, then

$$
r\left(f(y),\left\{f^{n}(x)\right\}\right) \leq r\left(y,\left\{f^{n}(x)\right\}\right),
$$

for each $y \in D$;

(4) by Proposition 4.1 , every $k_{D}$-bounded sequence $\left\{x_{n}\right\}$ in $D$ has a unique asymptotic center with respect to any nonempty, $k_{D}$-closed, and convex subset $C$ of $D$.

Corollary 4.3. Theorem 4.2 is valid for holomorphic self-mappings of $D$.

Proof. Each holomorphic self-mapping of $D$ is $k_{D}$-nonexpansive.

Remark 4.4. Note that in the case of the open unit ball $B_{H}$ of a Hilbert space $H$, the analogous theorem and corollary are known $[12,13,18,19]$.

Now, we study the structure of the fixed-point set of a holomorphic mapping. First, we recall two results from [5].

Lemma 4.5 ([5]). Let $X$ be a complex reflexive Banach space and $D$ a bounded strictly convex domain in $X$. If $f: D \rightarrow D$ is $k_{D}$-nonexpansive and has a fixed point, then $f$ has a fixed point in each nonempty, $f$-invariant, $k_{D}$-closed, and convex subset $C$ of $D$.

Theorem 4.6 (see [5]). Let $D$ be a bounded strictly convex domain in a complex reflexive Banach space $X$. If $f: D \rightarrow D$ is holomorphic $\left(k_{D}\right.$-nonexpansive), then $\operatorname{Fix}(f)$ is either empty or a holomorphic ( $k_{D}$-nonexpansive) retract of $D$.

In the case of a locally uniformly linearly convex metric space $\left(D, k_{D}\right)$, we have the following results - their proofs are practically the same of those given in [5] and they are based on the Bruck method $[1,2]$.

LeMmA 4.7. Let $X$ be a complex reflexive Banach space and $D$ a bounded convex domain in $X$ such that the metric space $\left(D, k_{D}\right)$ is locally uniformly linearly convex. If $f: D \rightarrow D$ is $k_{D}$-nonexpansive and has a fixed point, then $f$ has a fixed point in each nonempty, $f$-invariant, $k_{D}$-closed, and convex subset $C$ of $D$.

Theorem 4.8. Let $D$ be a bounded convex domain in a complex reflexive Banach space $X$ such that the metric space $\left(D, k_{D}\right)$ is locally uniformly linearly convex. If $f: D \rightarrow D$ is holomorphic ( $k_{D}$-nonexpansive), then $\operatorname{Fix}(f)$ is either empty or a holomorphic ( $k_{D}$-nonexpansive) retract of $D$.

\section{References}

[1] R. E. Bruck Jr., Nonexpansive retracts of Banach spaces, Bull. Amer. Math. Soc. 76 (1970), 384-386.

[2] _ Properties of fixed-point sets of nonexpansive mappings in Banach spaces, Trans. Amer. Math. Soc. 179 (1973), 251-262.

[3] M. Budzyńska, Domains which are locally uniformly linearly convex in the Kobayashi distance, to appear in Abstr. Appl. Anal. 
[4] - An example in holomorphic fixed point theory, to appear in Proc. Amer. Math. Soc.

[5] M. Budzyńska and T. Kuczumow, A strict convexity of the Kobayashi distance, Fixed Point Theory and Applications (Y. J. Cho, ed.), vol. 4, Nova Science Publishers, to appear.

[6] S. Dineen, The Schwarz Lemma, Oxford Mathematical Monographs, Oxford University Press, New York, 1989.

[7] S. Dineen, R. M. Timoney, and J.-P. Vigué, Pseudodistances invariantes sur les domaines d'un espace localement convexe, Ann. Scuola Norm. Sup. Pisa Cl. Sci. (4) 12 (1985), no. 4, 515-529 (French).

[8] C. J. Earle and R. S. Hamilton, A fixed point theorem for holomorphic mappings, Global Analysis (Proc. Sympos. Pure Math., Vol. 16, Berkeley, Calif., 1968), American Mathematical Society, Rhode Island, 1970, pp. 61-65.

[9] M. Edelstein, The construction of an asymptotic center with a fixed-point property, Bull. Amer. Math. Soc. 78 (1972), 206-208.

[10] T. Franzoni and E. Vesentini, Holomorphic Maps and Invariant Distances, Notas de Matemática, vol. 69, North-Holland Publishing, Amsterdam, 1980.

[11] K. Goebel and W. A. Kirk, Topics in Metric Fixed Point Theory, Cambridge Studies in Advanced Mathematics, vol. 28, Cambridge University Press, Cambridge, 1990.

[12] K. Goebel and S. Reich, Uniform Convexity, Hyperbolic Geometry, and Nonexpansive Mappings, Monographs and Textbooks in Pure and Applied Mathematics, vol. 83, Marcel Dekker, New York, 1984.

[13] K. Goebel, T. Sekowski, and A. Stachura, Uniform convexity of the hyperbolic metric and fixed points of holomorphic mappings in the Hilbert ball, Nonlinear Anal. 4 (1980), no. 5, 1011-1021.

[14] L. A. Harris, Schwarz-Pick systems of pseudometrics for domains in normed linear spaces, Advances in Holomorphy (Proc. Sem. Univ. Fed. Rio de Janeiro, Rio de Janeiro, 1977), North-Holland Math. Stud., vol. 34, North-Holland Publishing, Amsterdam, 1979, pp. 345-406.

[15] M. Jarnicki and P. Pflug, Invariant Distances and Metrics in Complex Analysis, de Gruyter Expositions in Mathematics, vol. 9, Walter de Gruyter, Berlin, 1993.

[16] S. Kobayashi, Invariant distances on complex manifolds and holomorphic mappings, J. Math. Soc. Japan 19 (1967), 460-480.

[17] _ Hyperbolic Manifolds and Holomorphic Mappings, Pure and Applied Mathematics, vol. 2, Marcel Dekker, New York, 1970.

[18] T. Kuczumow, Fixed points of holomorphic mappings in the Hilbert ball, Colloq. Math. 55 (1988), no. 1, 101-107.

[19] T. Kuczumow, S. Reich, and D. Shoikhet, Fixed points of holomorphic mappings: a metric approach, Handbook of Metric Fixed Point Theory (W. A. Kirk and B. Sims, eds.), Kluwer Academic Publishers, Dordrecht, 2001, pp. 437-515.

[20] T. Kuczumow and A. Stachura, Iterates of holomorphic and $k_{D}$-nonexpansive mappings in convex domains in $\mathbf{C}^{n}$, Adv. Math. 81 (1990), no. 1, 90-98.

[21] W. Rudin, Function Theory in the Unit Ball of $\mathbf{C}^{n}$, Grundlehren der Mathematischen Wissenschaften, vol. 241, Springer-Verlag, New York, 1980.

[22] J.-P. Vigué, La métrique infinitésimale de Kobayashi et la caractérisation des domaines convexes bornés [The Kobayashi infinitesimal metric and the characterization of bounded convex domains], J. Math. Pures Appl. (9) 78 (1999), no. 9, 867-876 (French). 
[23] Stricte convexité des domaines bornés et unicité des géodésiques complexes [Strict convexity of bounded domains and uniqueness of complex geodesics], Bull. Sci. Math. 125 (2001), no. 4, 297-310 (French).

Monika Budzyńska: Instytut Matematyki, Uniwersytet M. Curie-Sklodowskiej (UMCS), 20-031 Lublin, Poland; Instytut Matematyki Panstwowa Wyzsza Szkola Zawodowa, 20-120 Chełm, Poland

E-mail address: monikab@golem.umcs.lublin.pl 


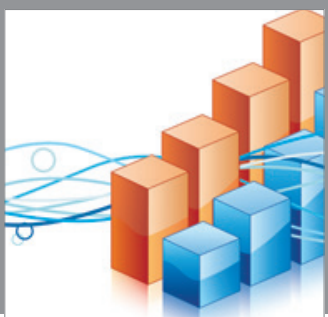

Advances in

Operations Research

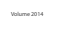

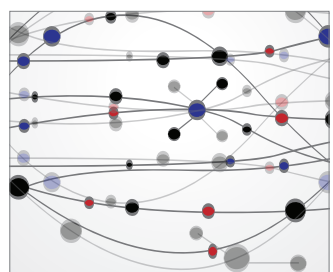

\section{The Scientific} World Journal
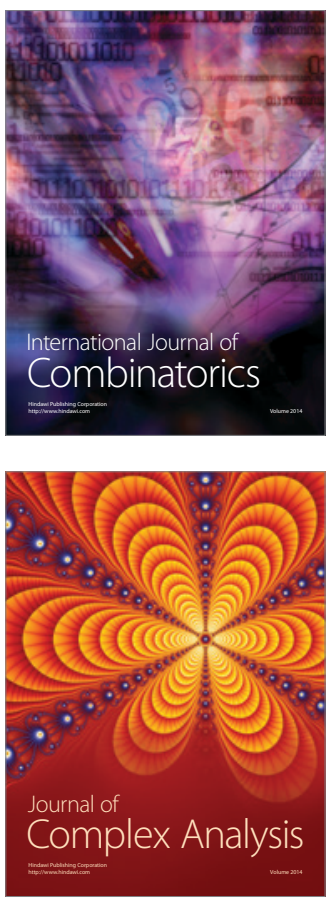

International Journal of

Mathematics and

Mathematical

Sciences
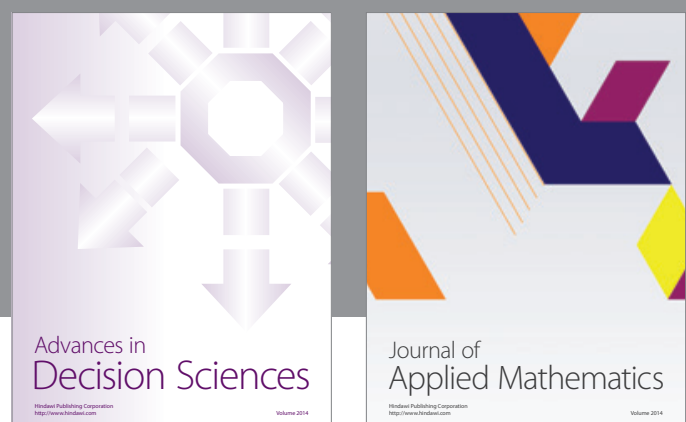

Journal of

Applied Mathematics
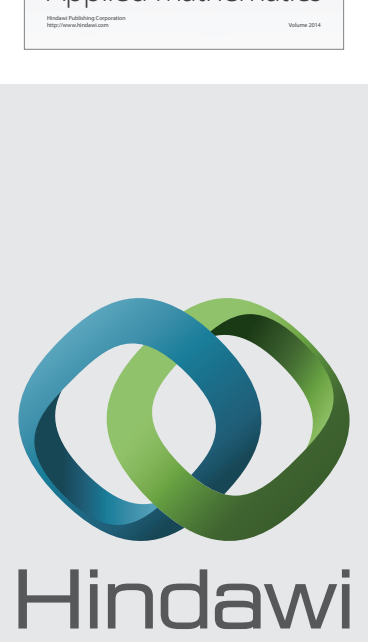

Submit your manuscripts at http://www.hindawi.com
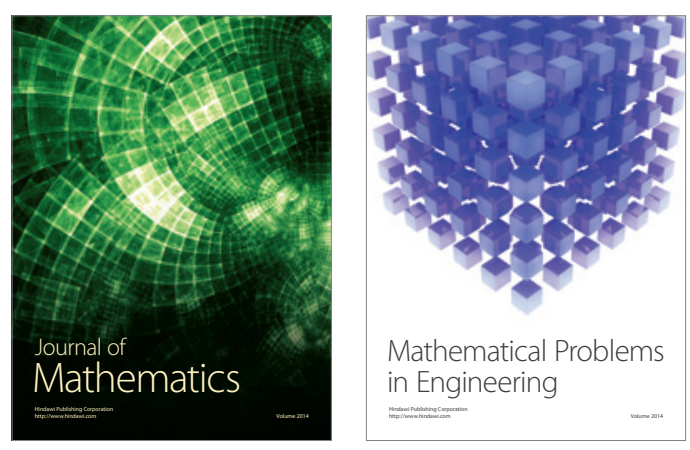

Mathematical Problems in Engineering
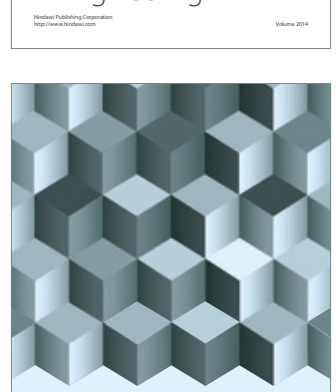

Journal of

Function Spaces
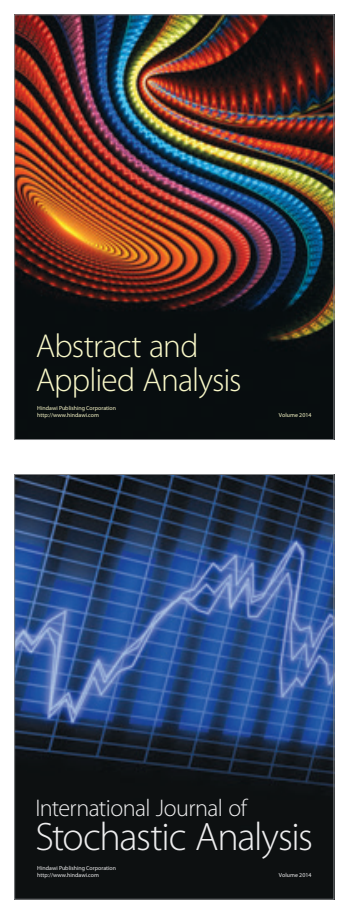

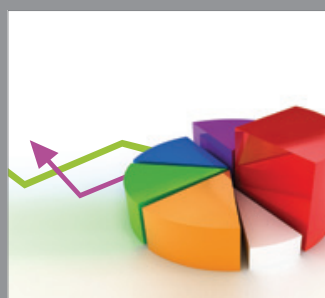

ournal of

Probability and Statistics

Promensencen
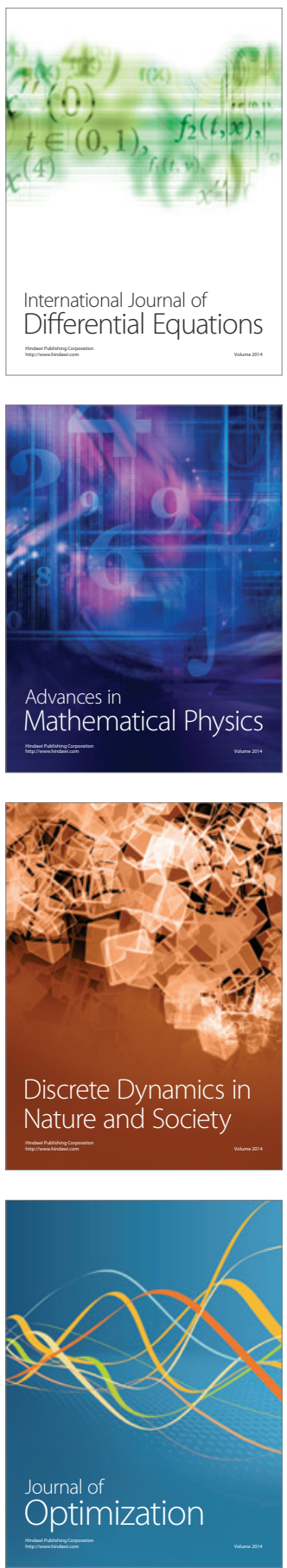